\title{
Cost Effectiveness and Budget Impact of Siponimod Compared to Interferon Beta-1a in the Treatment of Adult Patients with Secondary Progressive Multiple Sclerosis with Active Disease in Switzerland
}

\author{
Nadine Schur ${ }^{1} \cdot$ Kapil Gudala $^{2} \cdot$ Umakanth Vudumula $^{2} \cdot$ Sreelatha Vadapalle $^{2} \cdot$ Arjun Bhadhuri $^{1}$ (C) Alain Casanova $^{3}$. \\ Nicholas Adlard ${ }^{4} \cdot$ Matthias Schwenkglenks ${ }^{1}$
}

Accepted: 17 March 2021 / Published online: 1 April 2021

(c) The Author(s) 2021

\begin{abstract}
Objective The study aim was to evaluate the cost effectiveness and budget impact of siponimod compared to interferon beta-1a for adult patients with secondary progressive multiple sclerosis (SPMS) with active disease, from a Swiss health insurance perspective.

Methods We conducted an analysis using a Markov cohort model with a cycle length of 1 year, life-long time horizon, and discount rate of $3 \%$ for cost and health outcomes. We used a matching-adjusted indirect comparison to estimate clinical outcomes using data from the EXPAND randomised controlled trial of siponimod vs placebo and the Nordic SPMS randomised controlled trial of interferon beta-1a vs placebo as the basis for estimates of disability progression and relapse outcomes. We used 6-month confirmed disability progression results to estimate disability progression in the base-case analysis. We calculated quality-adjusted life-years (QALYs) based on an external study that administered the EQ-5D-3L questionnaire to European patients with multiple sclerosis. We included costs (Swiss Franc (CHF), year 2020) of drug acquisition/administration, adverse events and disease management. We also performed a budget impact analysis to estimate the cost over the first 3 years of introducing siponimod.

Results For the base case, siponimod resulted in mean incremental costs of CHF 84,901 (siponimod: CHF 567,838, interferon beta-1a: CHF 482,937) and mean incremental QALYs of 1.591 (siponimod: 7.495, interferon beta-1a: 5.905), leading to an incremental cost-effectiveness ratio of CHF 53,364 per QALY gained. In the probabilistic sensitivity analysis, the probability of the cost effectiveness of siponimod assuming a willingness-to-pay threshold of CHF 100,000 per QALY gained was 90\%. Siponimod was projected to result in drug administration costs for siponimod of CHF 23,817,856 in the first 3 years after introduction, accompanied by large cost offsets in drug acquisition of other multiple sclerosis drugs. Considering drug administration, monitoring and adverse event management costs, it was estimated to result in additional healthcare costs in Switzerland of CHF 2,177,021.

Conclusions In the base-case analysis, we found that siponimod may be cost effective for treating Swiss adult patients with SPMS with active disease. The results of the cost-effectiveness analyses are valid under the assumption that the efficacy of siponimod and the comparators on disability progression for the overall SPMS population would be the same in the active SPMS population.
\end{abstract}

Clinical Trial Identifier NCT01665144. This economic evaluation was based on the EXPAND trial.

Extended author information available on the last page of the article 


\section{Key Points}

Siponimod may be cost effective for treating Swiss adult patients with secondary progressive multiple sclerosis with active disease.

Related uncertainty is unavoidable given the lack of head-to-head trials directly comparing siponimod with other active treatments.

Siponimod is currently reimbursed as a treatment for active secondary progressive multiple sclerosis in Switzerland. In our budget impact analysis, it was projected to result in additional healthcare costs in Switzerland of Swiss Francs 2,177,021 in the first 3 years after introduction. A limitation of the budget impact analysis was not accounting for disease management and relapse costs.

\section{Introduction}

Multiple sclerosis (MS) is a chronic inflammatory and neurodegenerative disease of the central nervous system that leads to neurological impairment and severe disability [1]. It is characterised by damage to the myelin sheaths of neurons in the central nervous system and axonal loss, resulting in impaired brain, optic nerves and spinal cord function [1].

The majority of patients with MS present with a relapsing-remitting MS (RRMS) course of disease, which is characterised by acute episodes of neurological dysfunction, termed relapses, followed by complete or incomplete recovery and periods of clinical stability. Incomplete recovery from relapses leads to the accumulation of disability [2]. Over time, patients with RRMS may eventually transition to a secondary progressive course of disease (SPMS) in which they experience sustained progression of disability with or without superimposed relapses [3]. Population-based epidemiological studies to assess the prevalence of SPMS have reported a wide range between 7.5 and $41.5 \%$ of patients with MS across European countries [4, 5]. For Switzerland, reports range from 5.7 to $18 \%$ in the literature $[6,7]$ and physician feedback suggests that an equal number of patients (i.e. between 5.7 and 18\%) are in the transition phase from RRMS to SPMS. Secondary progressive MS can be further classified either into an active or non-active form. Patients with active SPMS experience relapses and/or show evidence of inflammatory activity in imaging, while patients with non-active disease do not [8].

There are several disease-modifying therapies (DMTs) currently used in Switzerland to treat SPMS. Interferon beta-1a (Avonex ${ }^{\circledR}$ produced by Biogen AG, or Rebif ${ }^{\circledR}$ produced by Merck Schweiz AG) has a first-line indication for relapsing SPMS, which excludes patients with active SPMS who only show evidence of inflammatory activity but no relapses. Interferon beta- $1 \mathrm{~b}$ (Betaferon ${ }^{\circledR}$ produced by Bayer AG) is indicated first line for active and nonactive SPMS. Ocrelizumab (Ocrevus ${ }^{\circledR}$ produced by Roche Pharma AG) is indicated for relapsing MS with active disease, which indirectly includes patients with SPMS who still have relapses and an active disease course [9]. Mitoxantrone (Novanton ${ }^{\circledR}$ produced by Meda Pharma GmbH) is indicated in SPMS as a second-line therapy for patients with rapidly progressing MS [10].

Siponimod (Mayzent ${ }^{\circledR}$ produced by Novartis Pharma AG) is a new DMT available for the first-line treatment of patients with active SPMS who do not have the genotype CYP2C $9 * 3 * 3$. Siponimod is administered as an oral tablet. Siponimod is a new member of a class of orally administered compounds, referred to as sphingosine1-phosphate receptor modulators. In patients with MS, siponimod prevents pathogenic effector lymphocytes from accessing their targets in the central nervous system [11-14]. In addition, siponimod may have neuroprotective effects by directly interfering with ongoing sphingosine1-phosphate-mediated astroglial inflammatory processes and/or stimulating sphingosine-1-phosphate 5-mediated pro-remyelination repair mechanisms [14-17]. In the EXPAND randomised controlled trial of 1652 patients with SPMS with active or non-active disease, siponimod was found, relative to placebo, to result in a statistically significant reduction in the risk of 6-month confirmed disability progression (CDP) [hazard ratio of 0.74 ; $95 \%$ confidence interval (CI) 0.60-0.92], and found to have a similar safety profile to other drugs in the class [18]. For the active SPMS subgroup, the hazard ratio for this reduction was estimated at 0.64 (95\% CI 0.47-0.86). Six-month CDP was defined as a 1-point increase in the Expanded Disability Status Scale (EDSS) score if the baseline EDSS score was 3.0-5.0, or a 0.5-point EDSS score increase if the baseline EDSS score was 5.5-6.5, and the increase in EDSS has to be confirmed at a scheduled visit at least 6 months apart [18]. It was also found in the EXPAND trial that siponimod, relative to placebo, resulted in a statistically significant reduction in the annualised relapse rate (rate ratio of 0.45 ; 95\% CI $0.34-0.59$; risk reduction $55 \%$ ) [18] for the overall EXPAND population, as well as for the active SPMS subgroup (rate ratio of $0.40 ; 95 \%$ CI $0.24-0.66$; risk reduction $60 \%$ ). Five-year follow-up results from the EXPAND trial have demonstrated that early initiation of siponimod treatment significantly reduces 6-month CDP and the rate of relapses for the overall SPMS population in the long term, as detailed in an abstract for the journal Neurology [19]. 
The aim of this study was to assess the cost effectiveness of a siponimod treatment strategy compared to interferon beta-1a treatment strategy, for adult patients with SPMS with active disease in Switzerland. We also assessed the budget impact of introducing siponimod for the treatment of active SPMS.

\section{Methods}

We used a Markov cohort model for our cost-effectiveness analysis, and an incremental cost per quality-adjusted lifeyear (QALY) gained and per life-year (LY) gained approach. We built and implemented the Markov cohort model using Microsoft Excel 2010. For the base-case analysis, we used a time horizon of age 100 years (interpreted as life-long), a cycle length of 1 year, and a discount rate of 3\% for costs and health outcomes (QALYs and LYs). Although there are no formal guidelines specifying what discount rate should be used for cost-utility analyses in Switzerland, a discount rate of $3 \%$ per year is generally accepted. We assessed costs from a Swiss health insurance system perspective for the main analysis and from an approximated societal perspective in a secondary analysis. We selected sources for Swiss unit costs such as national tariff lists and national statistical data based on institutional knowledge. We undertook a series of targeted literature searches on PubMed and Google to identify relevant local parameter values for the models (e.g. "cost multiple sclerosis Switzerland", "utility multiple sclerosis Switzerland").

\subsection{Model Structure and Population}

For the cost-effectiveness part, we implemented a Markov cohort model based on EDSS scores that measure the degree of disability in MS on a progressive scale from 0 (indicating normal neurological exam and no disability) to 10 (indicating death due to MS) (Fig. 1). During each model cycle, patients in an EDSS-based state could either remain in the same EDSS state, transition to a different EDSS state or transition to the death state. Patients who transition to a different EDSS state in a model cycle can either move to a better EDSS state (one or more steps) or a worse EDSS state (one or more steps). Besides transitioning between model states, in each model cycle, patients could also simultaneously experience a relapse event and/or discontinue DMT. Once a patient discontinued siponimod/interferon beta-1a treatment, this discontinuation was assumed to be permanent. Patients who discontinued treatment followed transitions of patients receiving best supportive care. During each 1-year cycle of the model, patients accrued costs and outcomes based on the state they had transitioned into from the previous cycle, as well as the occurrence of relapse events and whether they had already discontinued treatment.

Characteristics of the cohort at the start of the model were assumed to match the baseline characteristics of the

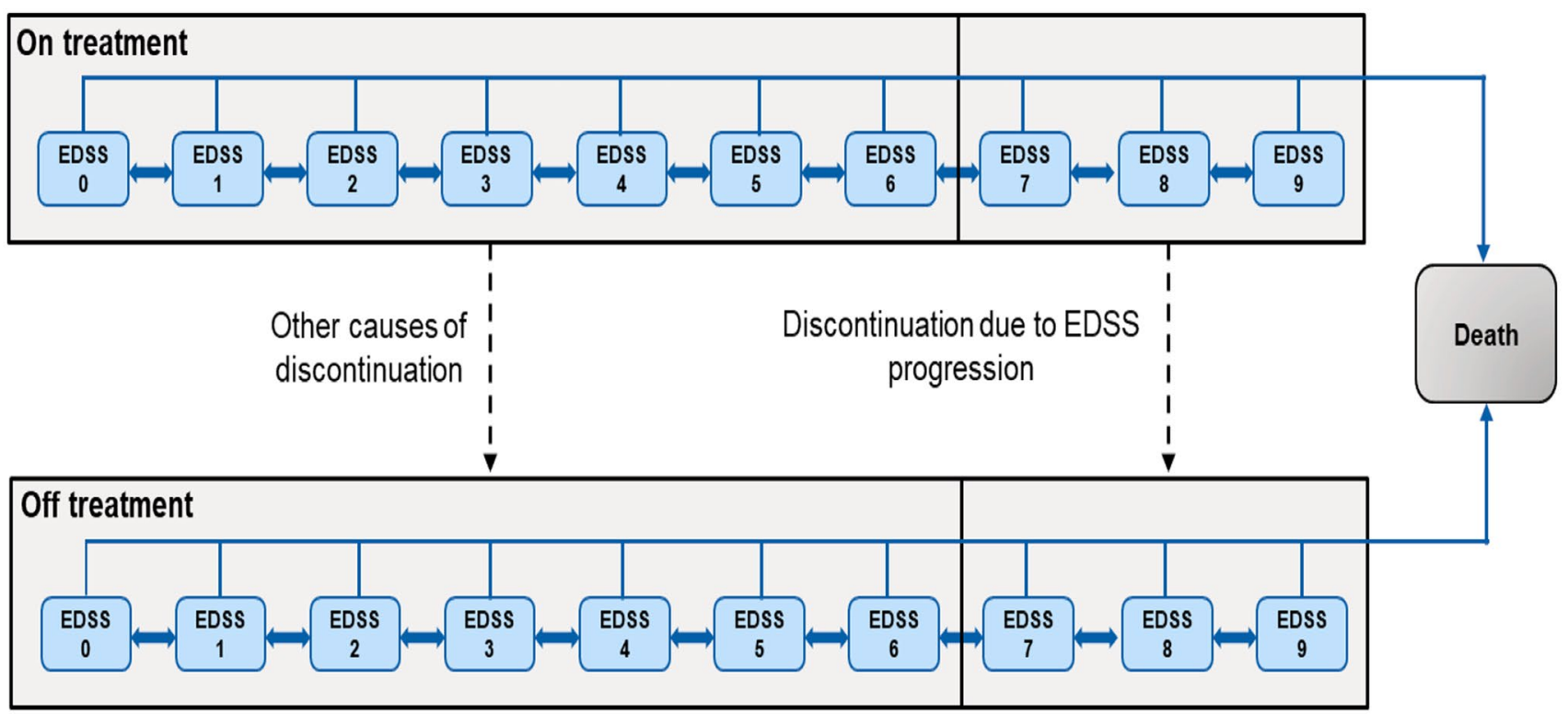

Fig. 1 Structure of the Markov model. A proportion of patients will remain in the same Expanded Disability Status Scale (EDSS) state at the end of a model cycle. Patients who transition to a different EDSS state in a model cycle can either move to a better EDSS state (one or more steps) or a worse EDSS state (one or more steps). Transition probabilities are specified in Appendix A1 of the ESM. In each of the EDSS states, patients may experience a relapse during a model cycle 
EXPAND trial participants with active SPMS. These characteristics were: having active SPMS, an EDSS score of $>1$ and $<8$, and not having the genotype CYP2C $9 * 3 * 3$. Furthermore, participants were $64 \%$ female, had a mean age of 46.6 years, mean weight of $70.3 \mathrm{~kg}$, mean time since MS diagnosis of 11.1 years, and mean time since SPMS diagnosis of 2.9 years.

\subsection{Intervention and Comparator}

The siponimod treatment strategy comprised siponimod and best supportive care (BSC). The comparator strategy comprised interferon beta-1a (Rebif $22 \mu \mathrm{g}$ dose three times a week) and BSC, which was selected as the comparator as interferon beta-1a is one of few first-line SPMS drugs for active disease approved in Switzerland. Published matching-adjusted indirect comparison (MAIC) results for this comparison were available [20]. In a scenario analysis, we used other approved SPMS drugs for active disease in Switzerland as the comparators (interferon beta- $1 \mathrm{~b}$ plus BSC; natalizumab plus BSC). Natalizumab (Tysabri ${ }^{\circledR}$, produced by Biogen AG) is not indicated for SPMS in Switzerland, but it was included as a comparator in a scenario analysis because it was tested in a recent clinical trial in an SPMS population that allowed MAIC, as reported by Samjoo et al. [20].

Siponimod (and BSC) is contraindicated for patients with genotype CYP2C $9 * 3 * 3$, administered as a daily $1-\mathrm{mg}$ dose for genotype CYP2C $9 * 2 * 3$ or $* 1 * 3$, and administered as a daily $2-m g$ dose for all other genotypes. Interferon beta- $1 \mathrm{a}$ is administered as a $22-\mu \mathrm{g}$ dose three times a week. Discontinuation of siponimod/interferon beta-1a was assumed to occur when the patient's EDSS score reaches seven or higher.

Background therapy in addition to siponimod or DMT is considered in the model as BSC and is assumed to be the same in both strategies. In this analysis, background therapies used for Swiss patients with MS included corticosteroids, symptomatic prescription drugs, over-the-counter drugs and other types of medication, as published by Calabrese et al. [21]

\subsection{Natural History}

We calculated transition probabilities between EDSS (and death) states used for the model based on data from the placebo arm of the EXPAND trial [18]. The transition probability matrix was generated through a multi-state model (MSM) approach (using the "MSM" package in R), in line with the natalizumab National Institute for Health and Care Excellence (NICE) manufacturer submission (TA127 [22]). A MSM was fitted for patients randomised to the placebo arm of the EXPAND trial by utilising information on the EDSS state at each scheduled visit, duration spent in each EDSS state and initial values of the transition intensity matrix. We use data from an observational dataset (the London Ontario MS dataset [23]) to calculate certain transition probabilities where it we identified the sample size in the EXPAND placebo arm was insufficient to reliably estimate [additional information on the method used to club EXPAND and London Ontario data are presented in Appendix A1 of the Electronic Supplementary Material (ESM)].

The MSM transition probability matrix derived from EXPAND data was validated by comparing patient distribution by EDSS state using MSM and EXPAND observed data after 1 and 2 years. We used data from participants with active SPMS in the EXPAND placebo arm as the basis for calculating underlying annual relapse rates for EDSS states 3-7. We assumed that the annual relapse rates for EDSS scores 8 and 9 would be the same as for an EDSS score of 7 , and used rate estimates from external studies for EDSS scores 0-2 [22, 24].

Background mortality was considered in the analysis by using age-specific and sex-specific mortality rates obtained from the Swiss Federal Statistical Office [25]. The excess mortality associated with MS was incorporated into the analysis using EDSS-specific mortality multiplication factors provided by Pokorski (Table 1) [26].

\subsection{Effectiveness Estimates}

All underlying transition probabilities are presented in Appendix A1 of the ESM, and were categorised to disability progression (transitions to a higher EDSS health state) and no disability progression (transitions to same or lower EDSS health state). We subsequently calculated treatmentadjusted transition probabilities for each EDSS-based health state (additional information on the method used to calculate treatment-adjusted transition probabilities are presented in Appendix A2 of the ESM). These were calculated based on the following formulae and applied in the model:

1. Treatment-adjusted disability progression $=1-(1-$ sum of all disability progression transition probabilities)^hazard rate of 6-month CDP for treatment vs placebo

2. Treatment-adjusted no disability progression $=1-$ treatment-adjusted disability progression

We incorporated hazard, relapse and discontinuation rates for the intervention and comparator strategies in the model. We estimated hazard and relapse rates for siponimod and interferon beta-1a using MAICs from the EXPAND and Nordic SPMS trials, respectively [20]. We used hazard rates for 6-month CDP to adjust the transition probabilities described in the previous section. We estimated these to be 0.48 for siponimod relative to placebo, 


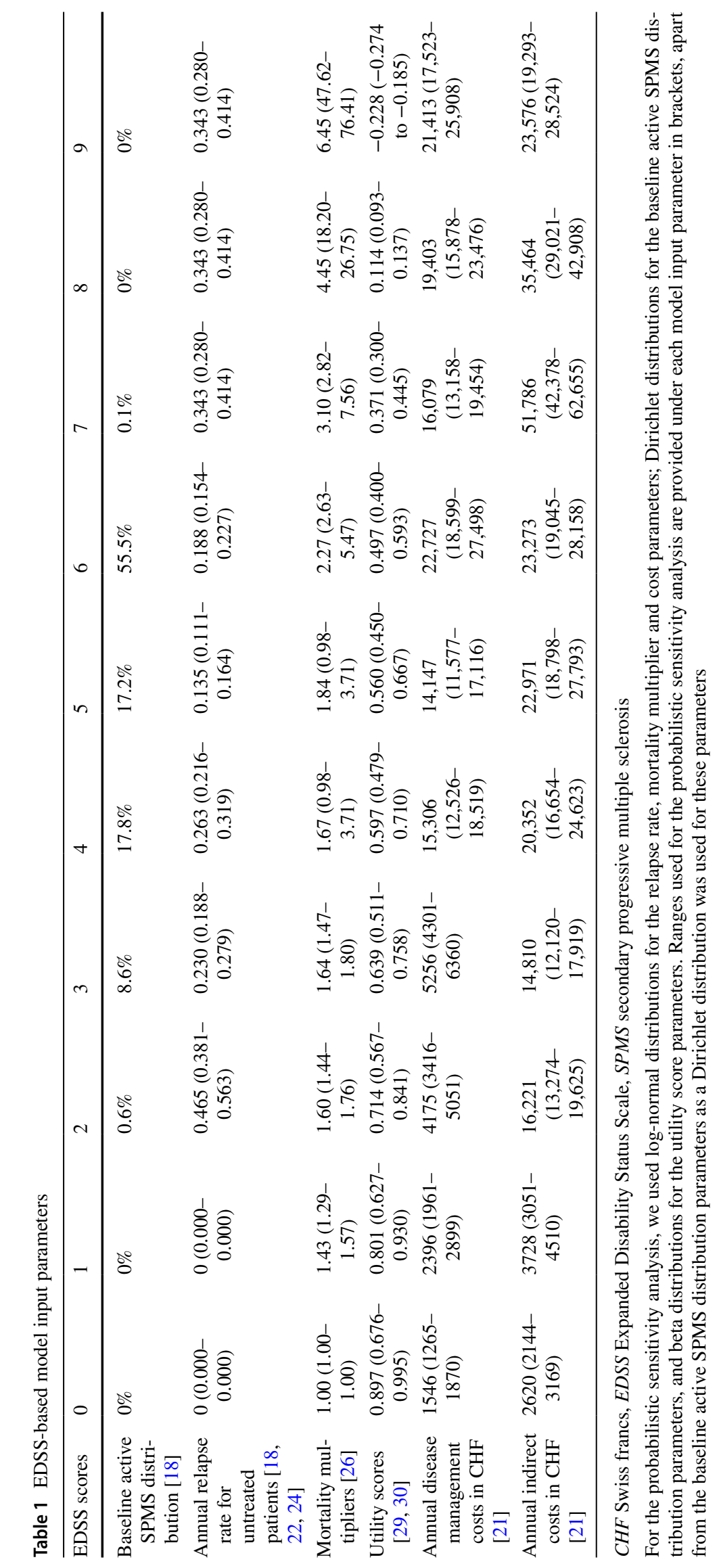


and 1.13 for interferon beta-1a relative to placebo. It was considered more appropriate to use 6-month CDP for the base-case analysis. As the time taken to recover from a relapse varies and people may not recover after a relapse after 3 months, 6-month CDP is used in clinical practice as a more robust measure of permanent disability progression compared to 3-month CDP [27].

The estimated annual relative risk of relapse was 0.50 for siponimod vs placebo and 0.69 for interferon beta1a vs placebo. Annual treatment discontinuation rates were assumed to be constant over time. For siponimod, it was estimated to be $6.37 \%$ based on EXPAND trial data (siponimod arm only) [18]. For interferon beta-1a, an annual treatment discontinuation rate of $9.16 \%$ was estimated by dividing the siponimod discontinuation rate of $6.37 \%$, by the relative risk of discontinuation for siponimod vs interferon beta- 1 a of 0.695 , which was estimated through an indirect treatment comparison using SPECTRIMIS trial data [28]. For scenario analyses that used alternative model comparators, the same procedure was adopted for obtaining effectiveness parameters; which resulted in estimates derived from the North American study for interferon beta-1b plus BSC, and from the ASCEND trial for natalizumab plus BSC (Table 2) [20].

\subsection{EQ-5D Scores}

We included European Quality of Life-5 Dimensions (EQ$5 \mathrm{D})$ scores in the analysis to represent health-related quality of life. Although utility values are available from participants of the EXPAND trial, these were not used because there were fewer or no patients with EDSS states 0, 1, 2, 8 and 9 , in the EXPAND trial. During each model cycle, patients were assigned an EQ-5D score based on their EDSS score, taken from a pooled sample of European patients with MS analysed by Kobelt et al. [29]. For the EQ-5D scores of patients who were alive, their EQ-5D score was subsequently inflated by 0.034 to adjust for the higher EQ-5D score of Swiss patients with MS relative to European patients with MS illustrated by Kobelt et al., and then reduced by 0.045 to adjust for the lower health status of patients with SPMS relative to general patients with MS as suggested by Orme et al. [30]. No further adjustment was incorporated with regard to active SPMS because of a lack of information. During each model cycle, a proportion of patients in each treatment strategy experienced a relapse or specific adverse event. The reduction in EQ-5D score associated with a relapse of 0.076 for a duration of 45 days was applied, obtained from an analysis of EXPAND trial data [18]. The reduction associated with each type of adverse event used for the model is specified in Table A3-2 of the ESM; primarily taken from a NICE technology appraisal for ocrelizumab [27].

\subsection{Resource Use and Cost Inputs}

All cost estimates obtained before 2020 were adjusted by inflation to 2020 values using the Swiss consumer price index [31].

Table 2 Model input parameters related to clinical effectiveness

\begin{tabular}{|c|c|c|c|}
\hline Modelled strategy & $\begin{array}{l}\text { MAIC-based hazard ratio of 6-month confirmed } \\
\text { disease progression vs placebo (range) [20] }\end{array}$ & $\begin{array}{l}\text { MAIC-based relative risk of relapse vs placebo } \\
\text { (range) [20] }\end{array}$ & $\begin{array}{l}\text { Treatment dis- } \\
\text { continuation rate } \\
\text { (range) }[18,28]\end{array}$ \\
\hline Siponimod and BSC & $\begin{array}{l}\text { Comparator }{ }^{\mathrm{a}} \text { is interferon beta-1a: } 0.48(0.22- \\
0.91) \\
\text { Comparator is interferon beta- } 1 \text { b: } 0.50(0.31- \\
0.76) \\
\text { Comparator is natalizumab: } 0.80(0.52-1.18)\end{array}$ & $\begin{array}{l}\text { Comparator }{ }^{\mathrm{a}} \text { is interferon beta-1a: } 0.50(0.28- \\
0.83) \\
\text { Comparator is interferon beta- } 1 \text { b: } 0.59(0.35- \\
0.93) \\
\text { Comparator is natalizumab: } 0.65(0.38-1.04)\end{array}$ & $6.37 \%(5.18-7.67)$ \\
\hline Comparator & $\begin{array}{l}\text { Comparator }{ }^{\mathrm{a}} \text { is interferon beta-1a: } 1.13(0.81- \\
1.54) \\
\text { Comparator is interferon beta- } 1 \mathrm{~b}: 0.92(0.70- \\
1.19) \\
\text { Comparator is natalizumab: } 1.06(0.72-1.50)\end{array}$ & $\begin{array}{l}\text { Comparator }{ }^{\mathrm{a}} \text { is interferon beta-1a: } 0.69(0.56- \\
0.84) \\
\text { Comparator is interferon beta- } 1 \text { b: } 0.65(0.47- \\
0.87) \\
\text { Comparator is natalizumab: } 0.45(0.32-0.63)\end{array}$ & $\begin{array}{l}\text { Comparator }^{\mathrm{a}} \\
\text { is interferon } \\
\text { beta-1a: } 9.16 \% \\
\text { (7.44-11.03) } \\
\text { Comparator } \\
\text { is interferon } \\
\text { beta-1b: } 7.33 \% \\
(5.96-8.82) \\
\text { Comparator is } \\
\text { natalizumab: } \\
6.29 \%(5.11- \\
7.58)\end{array}$ \\
\hline
\end{tabular}

$B S C$ best supportive care, MAIC matching-adjusted indirect comparison

${ }^{a}$ Base-case comparator is interferon beta-1a

${ }^{b}$ For the probabilistic sensitivity analysis, we used log-normal distributions for the hazard ratios and relative risk parameters, and beta distributions for the treatment discontinuation rate parameters. We used the ranges stated for the probabilistic sensitivity analysis 


\subsection{Drug Acquisition and Administration}

The price of siponimod used for the analysis was CHF 62.82 per dose. This was based on a calculation derived from the Spezialitätenliste of the weighted average of the prices of a 1-mg dose (price of CHF 62.69) and a 2-mg dose (price of CHF 62.83) [9], and assuming a genotype that $10 \%$ of patients would receive a 1-mg dose and $90 \%$ receive a $2-\mathrm{mg}$ dose. Siponimod is recommended to be administered daily until treatment is discontinued. The list price of interferon beta-1a was CHF 90.64 per 22- $\mu$ g dose, obtained from the Spezialitätenliste in April 2020, with patients with SPMS indicated to receive interferon beta- $1 \mathrm{a}$ as a $22-\mu \mathrm{g}$ dose three times per week until treatment is discontinued.

Besides the cost of acquiring siponimod and interferon beta-1a, we included the costs of DMT administration and monitoring for the period in which the patients were projected to receive DMT (Table 3). Full details are provided in Tables A4-1 and A4-2 of the ESM. We calculated annual DMT administration and monitoring costs based on the unit costs of the relevant resource items multiplied by the estimated number of required resource items per year. We estimated unit costs for outpatient physician visits, tests and imaging procedures from the Swiss tariff systems for outpatient physician services (TARMED) [32] and laboratory tests (Analysenliste) [33]. The Swiss clinical experts we consulted certified the validity of the assumptions we made about resource use items per year. The clinical experts were two neurologists based in Basel, Switzerland, who were each interviewed separately for $1 \mathrm{~h}$ each in 2019 . We showed the neurologists a list of estimated resource use items and values for patients with SPMS, and the neurologists amended this list based on their experience of how patients with SPMS are routinely treated in Switzerland.

\subsection{Disease Management Cost}

Costs of disease management in each model cycle were incurred based on the proportion of patients in each EDSS state multiplied by the annual disease management costs associated with the EDSS state. We obtained annual disease management costs assigned to each EDSS-based health state from a publication by Calabrese et al. of Swiss patients with MS (and assumed to be the same for patients with active SPMS owing to a lack of SPMS or active SPMS-specific data; as the exchange with the Swiss clinical experts did not cover this, we varied this set of parameters in a scenario analysis) [21]. As the set of disease management costs by Calabrese et al. incorporated relapse costs, we reduced each of the cost estimates by $23 \%$ to take out the relapse costs. This fixed percentage was estimated from the same study, and we used it across all EDSS categories as the authors only reported overall resource use costs with and without relapses rather than relapse costs for each EDSS category.

For the entire period in which patients were alive in the model, they were assumed to additionally receive BSC at an annual cost of CHF 1748.66. The annual BSC cost was again obtained from Calabrese et al. (and assumed to be the same for patients with active SPMS in our analysis) [21].

\subsection{Relapse Management}

The cost of managing active SPMS relapses was also incorporated into the analysis. We used an estimate based on Calabrese et al. of CHF 4344.34 for managing a relapse in Swiss patients with MS. For our analysis, we multiplied this estimate by two as according to a Swiss clinical expert we consulted, the cost of managing an SPMS relapse is approximately twice as high as managing a relapse in a general

Table 3 Model input parameters related to costs

\begin{tabular}{lll}
\hline Costs & Cost in CHF (range) & Source \\
\hline Siponimod drug cost (weighted average of 1-mg and 2-mg doses) & $62.82(50.26-75.38)$ & Spezialitätenliste [9] \\
Siponimod administration and monitoring cost year 1 & $1237.37(1012.58-1497.09)$ & Analysenliste [33] and TARMED [32] \\
Siponimod administration and monitoring cost year 2 & $260.03(212.79-314.60)$ & Analysenliste [33] and TARMED [32] \\
Interferon beta-1a drug cost (per 22- $\mu$ g dose) & $90.64(72.51-108.77)$ & Spezialitätenliste [9] \\
Interferon beta-1a administration and monitoring cost year 1 & $903.79(739.60-1,093.49)$ & Analysenliste [33] and TARMED [32] \\
Interferon beta-1a administration and monitoring cost year 2 & $286.76(234.67-346.95)$ & Analysenliste [33] and TARMED [32] \\
Annual best supportive care cost & $1748.66(1430.99-2115.69)$ & Calabrese et al. [21] \\
Genotype testing cost & $227.05(-)$ & Analysenliste [33] and TARMED [32] \\
Relapse management cost & $8689(7110-10,512)$ & Calabrese et al. [21], estimate doubled \\
& & based on a clinical expert
\end{tabular}

CHF Swiss francs

For the probabilistic sensitivity analysis, we used log-normal distributions for cost parameters. Drug acquisition and genotype testing costs were not varied in the probabilistic sensitivity analysis 
patient with MS. The clinical expert we consulted is a neurologist based in Basel, Switzerland.

\subsection{Adverse Event Costs}

We included the costs of serious and non-serious adverse events in the analysis (Table A3-3 of the ESM). We obtained the proportions of patients experiencing each adverse event from the EXPAND trial for siponimod [18] and from the NICE single technology appraisal of ocrelizumab for interferon beta-1a (Table A3-1 of the ESM) [27]. We multiplied each proportion by the associated unit costs of the adverse events, which we obtained from the Swiss DRG tariff data [34] for adverse events treated by hospitalisation, and from TARMED and Spezialitätenliste (i.e. the list of outpatient drugs reimbursed by the Swiss statutory health insurance) for adverse events treated by combined outpatient visits and prescription drug treatments $[9,32]$.

\subsection{Sensitivity Analysis}

We undertook one-way deterministic and probabilistic sensitivity analyses. In the one-way sensitivity analysis, we varied each cost, EQ-5D, and effectiveness parameter to the lower and upper bound of its $95 \% \mathrm{CI}$, to assess the resulting impact on the ICER, and presented the results of the 23 most influential parameters in a Tornado diagram. For siponimod and interferon beta-1a acquisition costs, given the absence of a 95\% CI for these, we applied a 20\% reduction and increase to these costs for the one-way sensitivity analyses. In the probabilistic sensitivity analysis, we generated 1000 simulated ICERs based on the mean value, standard error and distribution assigned to all of the model input parameters (excepting the discount rate, genotype testing costs, drug acquisition costs and the cut-off EDSS score of 7 for receiving DMT). If an empirically estimated standard error for a model input parameter did not exist, we assumed it was $10 \%$ of the mean parameter value. The distribution types assigned to input parameters were: log-normal for hazard ratios, relative risks (including relapse rates for untreated patients) and costs, beta for EQ-5D scores and treatment discontinuation rates, and Dirichlet for the EDSS-based baseline distribution of patients and the transition probability matrix.

To address uncertainty about various model assumptions and parameters, we undertook various scenario analyses. We explored natalizumab and interferon beta-1a as alternative model comparators. We varied discount rates and time horizons of the model. Instead of using MAIC estimates of treatment efficacy, we undertook a scenario analysis where we used direct estimates from trials (including for the active SPMS subgroup in the EXPAND trial [18]) without a matching adjustment. A further scenario analysis was undertaken where a 3-month CDP outcome instead of a 6-month CDP outcome from the EXPAND trial was used to model transitions between EDSS-based health states for the siponimod treatment strategy. We also undertook a scenario analysis using an (approximate) societal perspective that incorporated productivity losses due to short-term sick leave, and early retirement, which we extracted from Fig. 6 in the study by Calabrese et al. by digitalising the figure [21]. This analysis may be considered to be an approximation of a societal perspective rather than a full societal perspective, as we did not include informal carer costs and disutilities in this scenario analysis (or in any of the other analyses). In a further scenario analysis, we assumed that disease management costs are 1.5 times higher than were assumed for the base-case analysis.

\subsection{Budget Impact Analysis}

For the budget impact analysis, we adopted a 3-year time horizon and included costs of drug acquisition, drug administration and adverse events to estimate overall healthcare costs in Switzerland, comparing a situation where siponimod is approved with a situation where it is not approved. It was estimated that there are 1984 patients with active SPMS in Switzerland in 2021 (further details of how this was estimated are provided in Section A5 of the ESM). The distribution of patients across DMT classes (orals, injectables, infusions/no treatment), in the absence of siponimod, was estimated from an unpublished Novartis survey of 41 Swiss neurologists treating patients with MS. We recruited survey participants from office-based and hospital-based care settings and across the different language regions of Switzerland. We used commercially available IQVIA sales data for Switzerland from February 2020 to estimate the individual treatments within treatment classes obtained from the survey. For the situation with siponimod approved, we assumed that $8 \%$ of Swiss patients with active SPMS would be taking siponimod in year $1,17 \%$ in year 2 and $25 \%$ in year 3 . A full description of the methods for the budget impact analysis is provided in the ESM (Section A5).

\subsection{Model Validation}

We used a hybrid checklist of published checklists to validate the cost-effectiveness model. The model was found to be internally valid and externally valid. Internal validation of the model focused on the technical accuracy of the model and was intended to identify programming errors, data entry issues and logical inconsistencies in the model, which could then be corrected if identified. Therefore, we performed a variety of extensive tests before the model was used for the cost-effectiveness analysis. The aim of these tests was to demonstrate that the model was able to predict the results in a manner consistent with expectations. External validation 
of the model focused on the face validity and cross validity of results. The outcomes generated by an early version of the model using inputs from a previously published costeffectiveness analysis of fingolimod for the UK [35] were compared to the results from the fingolimod analysis. Face validity of the model was assessed by discussing the model results with two UK MS practicing physicians, via Skype interviews using a pre-specified list of questions.

\section{Results}

For the base-case analysis, we estimated that the siponimod treatment strategy generated discounted mean incremental drug acquisition costs of CHF 102,633 (siponimod: CHF 189,072, interferon beta-1a: CHF 86,438), mean incremental costs of the overall strategy of 84,901 (siponimod: CHF 567,838 , interferon beta-1a: CHF 482,937), mean incremental QALYs of 1.591 (siponimod: 7.495, interferon beta-1a: 5.905 ) and mean incremental LYs of 0.484 (siponimod: 18.896, interferon beta-1a: 18.412) (Table 4). This led to ICERs of CHF 53,364 per QALY gained and CHF 175,410 per LY gained. The siponimod treatment strategy was projected to result in mean incremental undiscounted LYs of 1.06 years (siponimod: 28.40, interferon beta-1a: 27.33). Higher drug acquisition costs in the siponimod strategy were to a limited extent compensated by lower disease management and relapse costs.

For the scenario analysis where we adopted a societal perspective, siponimod was projected to be a dominant treatment strategy (i.e. produce lower costs and higher QALYs than interferon beta-1a). For the analysis where the comparator was natalizumab (rather than interferon beta-1a), siponimod was estimated to be dominant. The scenario analysis where the primary endpoint used was 3 months of CDP (instead of 6 months) produced an ICER of CHF 136,514 per QALY gained. Full secondary analysis results are presented in Table 5.

In the one-way deterministic sensitivity analysis (Fig. 2), we varied over 150 parameters. For the majority of these, this resulted in only small changes to the base-case ICER. Varying the siponimod hazard ratio of 6-month CDP to the lower and upper bound of its $95 \%$ CI resulted in ICERs of CHF 34,448 and CHF 154,640, respectively. Varying the interferon beta-1a hazard ratio of 6-month CDP to the lower and upper bound of its $95 \%$ CI resulted in ICERs of CHF 74,540 and CHF 44,731, respectively. In the probabilistic sensitivity analysis, the siponimod treatment strategy was more cost effective than CHF 100,000 per QALY gained in $90 \%$ of simulations (Fig. 3a, b).

In the budget impact analysis, the introduction of siponimod for the treatment of active SPMS was projected to result in additional healthcare costs in Switzerland of $\mathrm{CHF}$ 2,177,021 in the first 3 years after introduction (CHF 308,342 in 2021, CHF 697,246 in 2022 and CHF 1,171,434 in 2023). The cost of CHF 2,177,021 over 3 years was generated from the estimated siponimod drug acquisition costs totalling to CHF 23,817,856, minus the displaced cost of acquiring other drugs totalling to $\mathrm{CHF} 19,179,912$, with some additional adjustments to account for reduced drug administration and monitoring costs and adverse event costs estimated from the market introduction of siponimod. Full budget impact results are documented in Appendix A5 of the ESM.
Table 4 Discounted base-case results (Swiss statutory health insurance perspective)

\begin{tabular}{llll}
\hline & $\begin{array}{l}\text { Siponimod and } \\
\text { BSC }\end{array}$ & $\begin{array}{l}\text { Interferon beta-1a and } \\
\text { BSC }\end{array}$ & Incremental \\
\hline Effectiveness parameters & & & \\
LYs & 18.896 & 18.412 & 0.484 \\
QALYs & 7.495 & 5.905 & 1.591 \\
Cost parameters (CHF) & & & \\
Total costs & 567,838 & 482,937 & 84,901 \\
Drug acquisition & 189,072 & 86,438 & 102,633 \\
Drug administration and monitoring & 2843 & 1821 & 1021 \\
Adverse events & 456 & 1010 & -555 \\
Disease management & 340,304 & 352,985 & $-12,681$ \\
Relapse & 35,165 & 40,682 & -5518 \\
Incremental cost-effectiveness ratio & & & 175,410 \\
Cost per (CHF) LY gained & & & 53,364 \\
Cost per (CHF) QALY gained & & & \\
\hline
\end{tabular}

$B S C$ best supportive care, $C H F$ Swiss Francs, $L Y$ life-years, $Q A L Y$ quality-adjusted life-years 
Table 5 Results of scenario analyses

Description of scenario

Incremental cost of siponi- Incremental QALYs ICER (CHF per mod strategy (CHF)

of siponimod

QALY gained)

Base-case analysis

$\begin{array}{lll}\text { CHF 84,901 } & 1.591 & \text { CHF 53,364 } \\ \text { CHF 117,916 } & 2.540 & \text { CHF 46,422 } \\ \text { CHF 93,673 } & 1.841 & \text { CHF 50,882 } \\ \text { CHF 66,565 } & 1.082 & \text { CHF 61,546 } \\ \text { CHF 33,284 } & 0.200 & \text { CHF 166,667 } \\ \text { CHF 52,988 } & 0.548 & \text { CHF 96,623 } \\ \text { CHF 71,671 } & 1.152 & \text { CHF 62,199 } \\ \text { CHF 84,900 } & 1.591 & \text { CHF 53,364 } \\ \text {-CHF 11,770 } & 1.59 & \text { Dominant } \\ \text { CHF 67,814 } & 0.497 & \text { CHF 136,514 } \\ \text { CHF 76,751 } & 1.076 & \text { CHF 71,326 } \\ \text {-CHF 101,967 } & 0.598 & \text { Dominant } \\ \text { CHF 74,939 } & 1.192 & \text { CHF 62,872 } \\ \text { CHF 78,560 } & 1.591 & \text { CHF 49,378 }\end{array}$

Discount rate: $0 \%$ for costs and outcomes

Discount rate: $2 \%$ for costs and outcomes

Discount rate: $6 \%$ for costs and outcomes

Time horizon: 5 years

Time horizon: 10 years

Time horizon: 20 years

Time horizon: 50 years

Societal perspective

Primary endpoint is 3 months of confirmed disability progression

Non-MAIC-based effectiveness

Alternative model comparator assumed: natalizumab

Alternative model comparator assumed: interferon beta-1b

Disease management costs were 1.5 times higher than in the base case

CHF 78,560

1.591

CHF 49,378

CHF Swiss Francs, ICER incremental cost-effectiveness ratio, MAIC matching-adjusted indirect comparison, $Q A L Y s$ quality-adjusted life-years

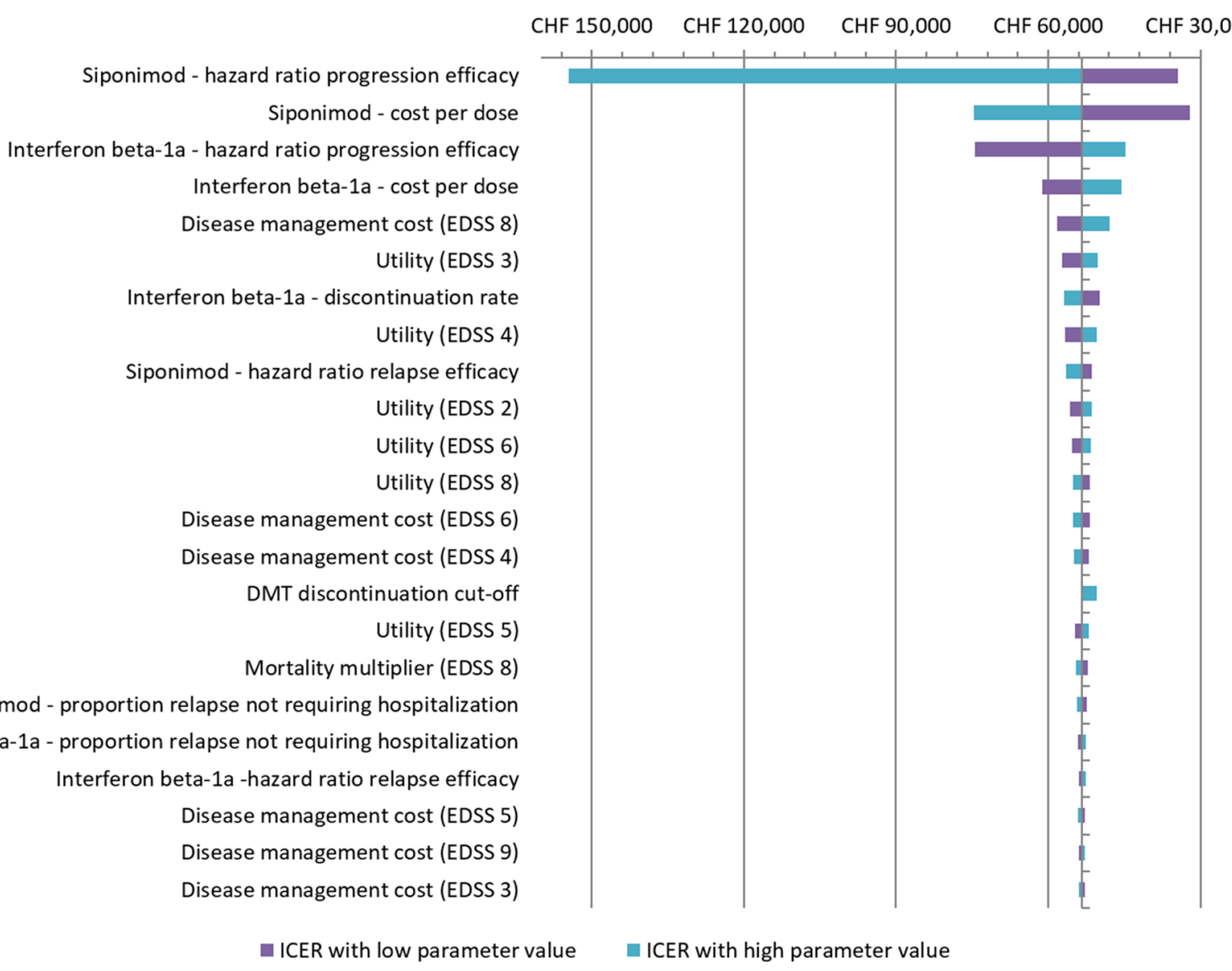

Fig. 2 Tornado diagram of deterministic sensitivity analysis results: the most influential model parameters. The cost effectiveness of the siponimod strategy vs the interferon beta-1a strategy is assessed in the figure. The base-case incremental cost-effectiveness ratio (ICER) of Swiss Francs (CHF) 53,364 per quality-adjusted life-year gained is represented by the vertical line in the graph. Additional information on the parameters and their respective bounds is provided in Table A6 of the ESM. DMT disease-modifying therapy, EDSS Expanded Disability Status Scale 


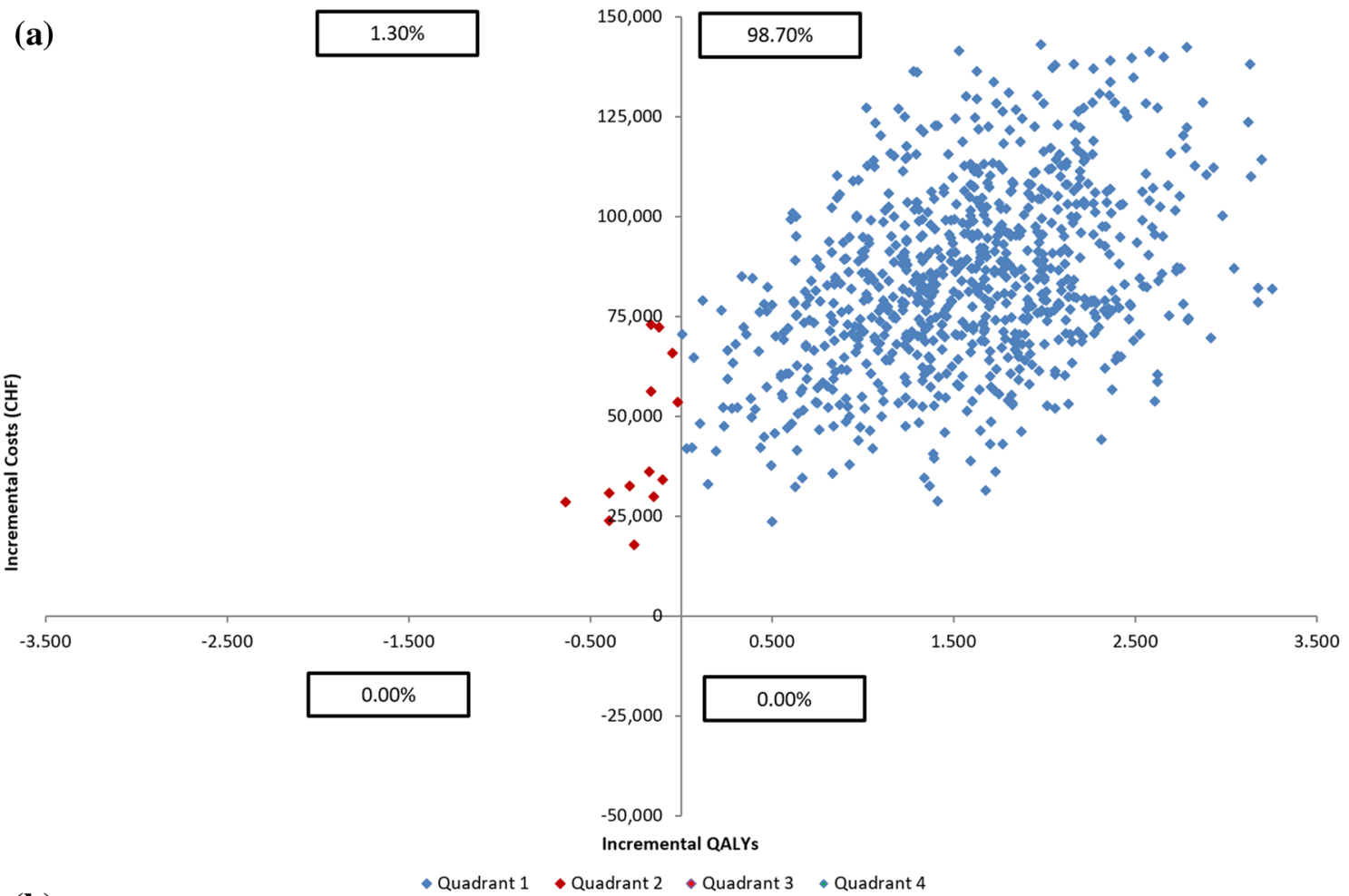

(b)

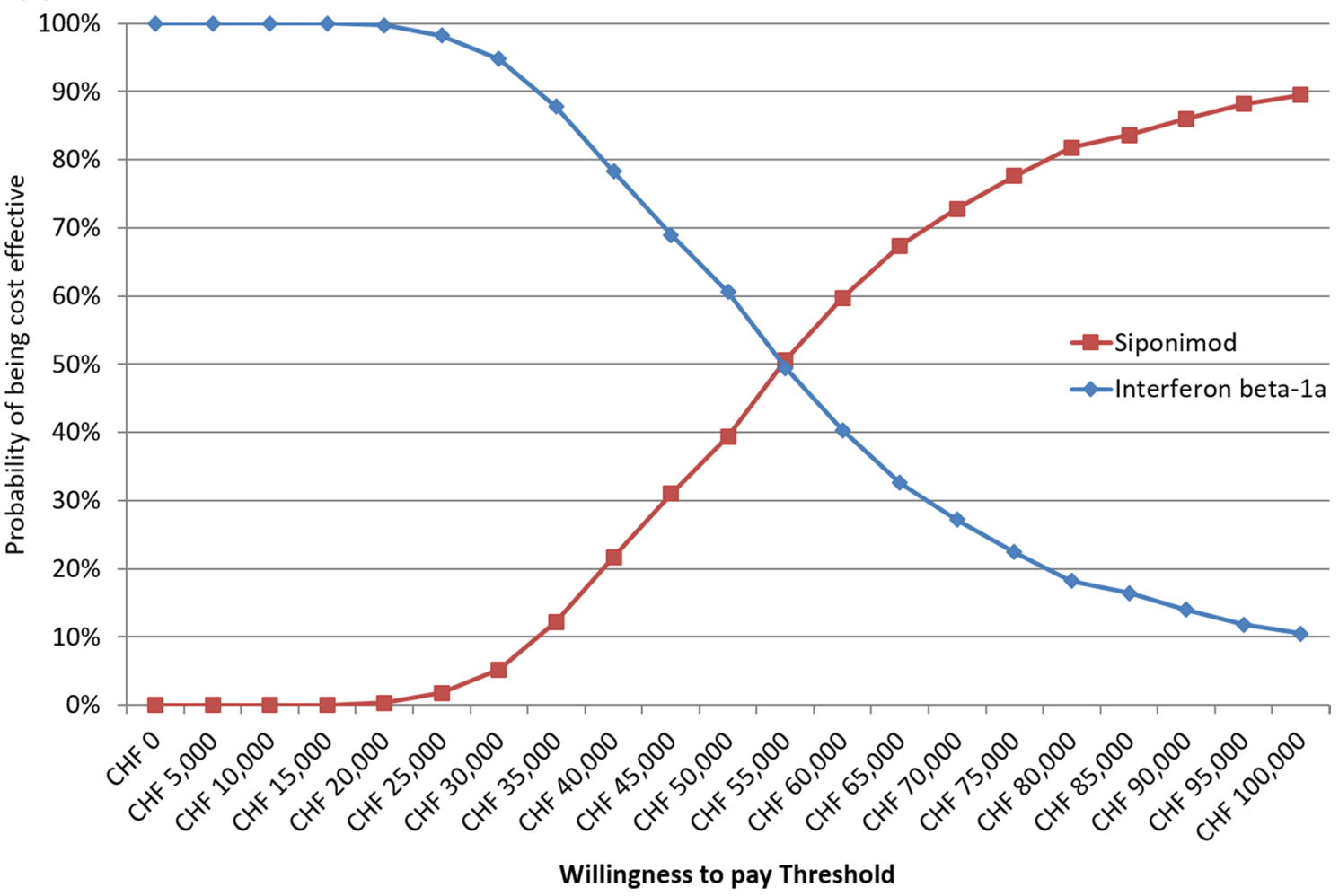

Fig. 3 Cost-effectiveness plane (a) and acceptability curve (b). CHF Swiss Francs, QALYs quality-adjusted life-years 


\section{Discussion}

The cost effectiveness of siponimod has previously been researched and published in the USA [8], the UK [28] and Canada [36], and has been approved for use in adult patients with SPMS with active disease in all three settings. In the USA and Canada, it was found that siponimod was unlikely to be a cost-effective treatment for adult patients with SPMS with active disease at the list prices that were originally proposed for these countries. The assessment for the USA was based on a much higher proposed price of siponimod than in this analysis. In the UK, siponimod was considered likely to be cost effective, and was approved by NICE following a confidential price reduction to the siponimod list price. Our research found siponimod is likely to be cost effective in Switzerland when priced at CHF 62.69 per 1-mg dose and CHF 62.83 per 2-mg dose, if assuming a cost-effectiveness threshold of CHF 100,000 per QALY gained, as it produced an ICER of CHF 53,364 per QALY gained. Although there are no formal guidelines specifying what threshold should be used for cost-utility analyses in Switzerland, a threshold of CHF 100,000 per QALY gained has been considered in previously published analyses. We found that an ICER below CHF 100,000 per QALY gained was maintained in the majority of sensitivity and scenario analyses undertaken. Our base-case analysis used CDP at least 6 months after the previous assessment (6-month CDP), as the basis for estimating the efficacy of siponimod for the cost-effectiveness analysis. Confirmed disability progression after 3 months (which was the primary outcome of the EXPAND trial) is considered a less robust efficacy measure of permanent disability progression than progression confirmed after 6 months, as the time taken to recover from a relapse varies and people may not recover from a relapse after 3 months. Furthermore, longer observation periods are preferable over shorter periods, as they reduce the uncertainties arising from extrapolation to the time horizons required for a cost-effectiveness analysis, provided there is no substantially increased risk of attrition bias. There was no difference in sample size between the 3-month CDP and 6-month CDP outcomes in the EXPAND trial for siponimod, indicating no additional risk of attrition bias as a result of using 6-month CDP. Six-month CDP was the only CDP outcome that was measured in the Nordic SPMS trial for interferon beta-1a. When we adopted the approach of using CDP after 3 months in a scenario analysis, a much higher ICER of CHF 136,514 per QALY gained for siponimod was estimated.

There were several limitations to our study. Effects were seen during a limited clinical trial observation period, and were applied in the long term. This implies an assumption of continued treatment effect. Disability progression transition probabilities between EDSS levels do not reflect transition probabilities between EDSS levels that changed as a result of confirmed disability progression. A similar limitation is also applicable to the previous cost-effectiveness models in the literature. The comparator for the base-case cost-effectiveness analysis we performed was interferon beta-1a, although there are other comparators that may be used in Switzerland for patients with SPMS such as interferon beta-1b and natalizumab, which we compared with siponimod in scenario analyses. Furthermore, fingolimod was not used as a comparator in the analysis, as although it is a frequently used treatment for MS in Switzerland, a relevant trial for fingolimod for SPMS was not identified in the systematic review by Samjoo et al. [20]. The EXPAND trial recruited patients with baseline EDSS scores from 3.0 to 6.5, thus our results may not generalise to patient with active SPMS outside of this range of EDSS scores. We estimated the clinical effectiveness of interferon beta-1a based on data from a single randomised controlled trial that reported no effect of interferon beta-1a on disability progression [37]. However, other randomised controlled trials have found slightly more positive evidence of a reduction in disability progression from treatment with beta- $1 \mathrm{a}$ or beta- $1 \mathrm{~b}$ interferons [38]. To address this limitation, we carried out a scenario analysis using interferon beta- $1 \mathrm{~b}$ as a comparator. Because of the absence of available MAIC results for active SPMS, we used hazard ratios for 6-month CDP and relative risks of relapse estimated for patients with SPMS regardless of subtype, and assumed these were applicable to the active SPMS subtype. To address the limitation, we undertook a scenario analysis using results directly reported from the EXPAND trial of participants with active SPMS and without a matching adjustment [18], which has the limitation that differences in the baseline characteristics of the study populations are not accounted for. This approach produced a slightly less favourable ICER of CHF 71,326 per QALY gained. In the budget impact analysis, there was an inherent uncertainty about market uptake over the first 3 years. The size of the active SPMS population is subject to uncertainty because of the nature of the disease. Patients gradually transition from RRMS to SPMS, limiting exact estimates of the number of patients. The diagnosis of SPMS/active SPMS is hindered by the relatively small number of DMTs approved in this indication as opposed to RRMS. Consequently, neurologist feedback was required to estimate DMT use in the siponimod target population. Nevertheless, these limitations are not expected to strongly affect the budget impact of the introduction of siponimod as a high rate of patients with MS currently receive DMT and remain taking treatment when transitioning to SPMS. It is thus expected that a large proportion of patients would transition from a currently reimbursed DMT to Siponimod, resulting in budget offsets. 


\section{Conclusions}

Our base-case analysis indicates that siponimod may be a cost-effective treatment for patients with active SPMS. Related uncertainty of all the results provided is unavoidable given the lack of head-to-head trials directly comparing siponimod with other active treatments. The results of the cost-effectiveness analyses are valid under the assumption that the efficacy of siponimod and the comparators on disability progression for the overall SPMS population would be the same in the active SPMS population. Siponimod is expected to replace different treatments with indications in RRMS and SPMS, offsetting a large proportion of the siponimod costs. This indicates siponimod may be affordable to healthcare payers in Switzerland.

Supplementary Information The online version contains supplementary material available at https://doi.org/10.1007/s40273-021-01023-8.

Acknowledgements We thank Prof. Dr. Ludwig Kappos and Dr. Johannes Lorscheider who provided extensive clinical expertise to verify the validity of the clinical inputs for the analysis. We also thank Dr. C. Simone Sutherland for her help in obtaining clinical inputs, and Vivek Khurana for providing guidance.

\section{Declarations}

Funding This study was funded by Novartis Pharma Schweiz AG, including research funding received by Matthias Schwenkglenks via Universität Basel. Open Access funding was provided by Universität Basel (Universitätsbibliothek Basel).

Conflict of interest Alain Casanova is an employee of Novartis Pharma Schweiz AG, Nicholas Adlard is an employee of Novartis Pharma AG, and Kapil Gudala, Umakanth Vudumula and Sreelatha Vadapalle are employees of Novartis Healthcare Private Limited. Matthias Schwenkglenks received research funding from Novartis Pharma Schweiz AG, via Universität Basel.

Ethics approval No specific ethical approval was obtained for this study, as the present economic model did not involve the recruitment of human or animal subjects or the collection of research samples.

Consent to participate Not applicable.

Consent for publication Not applicable.

Availability of data and material A request for the Excel models can be made to the corresponding author but is not guaranteed because of copyright. Acceptance of the request is subject to approval from Novartis Pharma AG.

Code availability Not applicable.

Author contributions AC, NS and MS conceptualised the study. KG, $\mathrm{NA}$, and UV produced the underlying cost-effectiveness model. KG, NA and SV produced the underlying budget impact model. NS completed the cost-effectiveness adaptation and $\mathrm{AB}$ completed the budget impact adaptation. NS and AB drafted the manuscript. AC organised and led meetings with Swiss clinical experts, provided methodological guidance to estimate parameters and undertook primary research to estimate market shares in Switzerland. All authors contributed to interpreting the results and critically reviewed and edited the manuscript.

Open Access This article is licensed under a Creative Commons Attribution-NonCommercial 4.0 International License, which permits any non-commercial use, sharing, adaptation, distribution and reproduction in any medium or format, as long as you give appropriate credit to the original author(s) and the source, provide a link to the Creative Commons licence, and indicate if changes were made. The images or other third party material in this article are included in the article's Creative Commons licence, unless indicated otherwise in a credit line to the material. If material is not included in the article's Creative Commons licence and your intended use is not permitted by statutory regulation or exceeds the permitted use, you will need to obtain permission directly from the copyright holder. To view a copy of this licence, visit http://creativecommons.org/licenses/by-nc/4.0/.

\section{References}

1. Compston A, Coles A. Multiple sclerosis. Lancet. 2008;372(9648):1502-17.

2. National Multiple Sclerosis Society. Types of MS: relapsingremitting MS; 2019. https://www.nationalmssociety.org/Whatis-MS/Types-of-MS/Relapsing-remitting-MS (Accessed $20 \mathrm{Mar}$ 2021).

3. Tremlett H, Yinshan Z, Devonshire V. Natural history of secondary-progressive multiple sclerosis. Mult Scler. 2008;14(3):314-24.

4. OFSEP. Description of OFSEP cohort; 2018. http://www.ofsep. org/fr/la-cohorte-ofsep/descriptif-de-la-cohorte (Accessed $20 \mathrm{Mar}$ 2021).

5. Khurana V, Sharma H, Medin J. Estimated prevalence of secondary progressive multiple sclerosis in the USA and Europe: results from a systematic literature search. Neurology. 2018;90(15):4.

6. Puhan MA, Steinemann N, Kamm CP, Muller S, Kuhle J, Kurmann $\mathrm{R}$, et al. A digitally facilitated citizen-science driven approach accelerates participant recruitment and increases study population diversity. Swiss Med Wkly. 2018;148:w14623.

7. Calabrese M, Poretto V, Favaretto A, Alessio S, Bernardi V, Romualdi $\mathrm{C}$, et al. Cortical lesion load associates with progression of disability in multiple sclerosis. Brain. 2012;135(Pt 10):2952-61.

8. Institute for Clinical and Economic Review. Siponimod for the treatment of secondary progressive multiple sclerosis: effectiveness and value; 2019.

9. Federal Office of Public Health. Spezialitätenliste (SL); 2019. http://www.spezialitaetenliste.ch (Accessed 20 Mar 2021).

10. Compendium.ch ${ }^{\circledR}$. https://compendium.ch/ (Accessed $20 \mathrm{Mar}$ 2021).

11. Gergely P, Nuesslein-Hildesheim B, Guerini D, Brinkmann V, Traebert M, Bruns C, et al. The selective sphingosine 1-phosphate receptor modulator BAF312 redirects lymphocyte distribution and has species-specific effects on heart rate. Br J Pharmacol. 2012;167(5):1035-47.

12. Bar-Or A, Derfuss T, Vermersch P, editors. Longitudinal changes in lymphocyte subsets of siponimod-treated patients with SPMS. 7th Joint European Committee for Treatment and Research in Multiple Sclerosis-Americas Committee for Treatment and Research in Multiple Sclerosis Meeting, 25-28 October 2017, Paris. 
13. Sarr C, Savelieva M, Ette G, editors. Modeling dose-PK-lymphocytes relationship under siponimod (BAF312) treatment to infer time to immune reconstitution [poster]. Presented at the 2014 Joint Americas Committee for Treatment and Research in Multiple Sclerosis-European Committee for Treatment and Research in Multiple Sclerosis Meeting, 10-13 September 2014; Boston (MA).

14. Bigaud M, Nuesslein-Hildesheim B, Tran T, editors. Siponimod is a functional agonist for the S1P5 receptor [poster]. Presented at the 70th Annual American Academy of Neurology Annual Meeting, 21-27 April 2018, Los Angeles (CA).

15. Gentile A, Musella A, Bullitta S, Fresegna D, De Vito F, Fantozzi $\mathrm{R}$, et al. Siponimod (BAF312) prevents synaptic neurodegeneration in experimental multiple sclerosis. J Neuroinflammation. 2016;13(1):207.

16. Zalc B, editor. Impact of siponimod (BAF 312) on CNS remyelination in a transgenic Xenopus model [poster]. Presented at the 2014 Joint Americas Committee for Treatment and Research in Multiple Sclerosis-European Committee for Treatment and Research in Multiple Sclerosis Meeting, 10-13 September 2014, Boston (MA).

17. Deogracias R, Schubart A, Barde Y-A, editors. Siponimod increases brain-derived neurotrophic factor (BDNF) levels in cortical neuronal cultures and naïve healthy mice [poster]. Presented at the 34th Congress of the European Committee for Treatment and Research in Multiple Sclerosis, 10-12 October 2018, Berlin.

18. Kappos L, Bar-Or A, Cree BAC, Fox RJ, Giovannoni G, Gold R, et al. Siponimod versus placebo in secondary progressive multiple sclerosis (EXPAND): a double-blind, randomised, phase 3 study. Lancet. 2018;391(10127):1263-73.

19. Kappos L, Giovannoni G, Gold R, Fox RJ, Vermersch P, Benedict $\mathrm{RH}$, et al. Long-term efficacy and safety of siponimod in patients with SPMS: EXPAND extension analysis up to 5 years (4128). AAN Enterprises; 2020.

20. Samjoo IA, Worthington E, Haltner A, Cameron C, Nicholas $\mathrm{R}$, Rouyrre N, et al. Matching-adjusted indirect treatment comparison of siponimod and other disease modifying treatments in secondary progressive multiple sclerosis. Curr Med Res Opin. 2020;36(7):1157-66.

21. Calabrese P, Kobelt G, Berg J, Capsa D, Eriksson J. European Multiple Sclerosis Platform. New insights into the burden and costs of multiple sclerosis in Europe: results for Switzerland. Mult Scler. 2017;23(2 Suppl):192-203.

22. National Institute for Health and Care Excellence (NICE). Natalizumab for the treatment of adults with highly active relapsingremitting multiple sclerosis; 2007. https://www.nice.org.uk/guida nce/ta127/history (Accessed 20 Mar 2021).

23. Mauskopf J, Fay M, Iyer R, Sarda S, Livingston T. Cost-effectiveness of delayed-release dimethyl fumarate for the treatment of relapsing forms of multiple sclerosis in the United States. J Med Econ. 2016;19(4):432-42.
24. Patzold U, Pocklington PR. Course of multiple sclerosis: first results of a prospective study carried out of 102 MS patients from 1976-1980. Acta Neurol Scand. 1982;65(4):248-66.

25. Swiss Federal Office of Statistics. Geburten und Todesfälle; 2018. https://www.bfs.admin.ch/bfs/de/home/statistiken/bevoelkerung/ geburten-todesfaelle.html (Accessed 20 Mar 2021).

26. Pokorski RJ. Long-term survival experience of patients with multiple sclerosis. J Insur Med. 1997;29(2):101-6.

27. National Institute for Health and Care Excellence (NICE). Ocrelizumab for treating relapsing multiple sclerosis [TA533]; 2018. https://www.nice.org.uk/guidance/indevelopment/gid-ta10152/ documents (Accessed 20 Mar 2021).

28. Secondary progressive efficacy clinical trial of recombinant interferon-beta-1a in MSSG. Randomized controlled trial of interferon-beta-1a in secondary progressive MS: clinical results. Neurology. 2001;56(11):1496-504.

29. Kobelt G, Thompson A, Berg J, Gannedahl M, Eriksson J, Group MS, et al. New insights into the burden and costs of multiple sclerosis in Europe. Mult Scler. 2017;23(8):1123-36.

30. Orme M, Kerrigan J, Tyas D, Russell N, Nixon R. The effect of disease, functional status, and relapses on the utility of people with multiple sclerosis in the UK. Value Health. 2007;10(1):54-60.

31. Swiss Federal Office of Statistics. Landesindex der Konsumentenpreise; 2019. https://www.bfs.admin.ch/bfs/de/home/statistiken/ preise/landesindex-konsumentenpreise.html (Accessed 20 Mar 2021).

32. FMH Swiss Medical Association. TARMED-Tarifbrowser Version 01.09.00_BR_KVG; 2018. https://www.fmh.ch/ambulante_tarife/ tarmed-tarif/tarmed_tarifbrowser-datenbank.html (Accessed 20 Mar 2021).

33. Swiss Federal Office of Public Health. Analysenliste Version vom 01.03.2019; 2019. https://www.bag.admin.ch/bag/de/home/versi cherungen/krankenversicherung/krankenversicherung-leistungentarife/Analysenliste.html (Accessed 20 Mar 2021).

34. Datenspiegel SwissDRG 6.0; 2019. https://datenspiegel60.swiss drg.org/ (Accessed 20 Mar 2021).

35. National Institute for Health and Care Excellence (NICE). Fingolimod for the treatment of highly active relapsing-remitting multiple sclerosis; 2012. https://www.nice.org.uk/guidance/ta254 (Accessed 20 Mar 2021).

36. National Institute for Health and Care Excellence, 2020. Siponimod for treating secondary progressive multiple sclerosis. https:// www.nice.org.uk/guidance/TA656 (Accessed 8 Dec 2020).

37. Andersen O, Elovaara I, Farkkila M, Hansen HJ, Mellgren SI, Myhr KM, et al. Multicentre, randomised, double blind, placebo controlled, phase III study of weekly, low dose, subcutaneous interferon beta-1a in secondary progressive multiple sclerosis. J Neurol Neurosurg Psychiatry. 2004;75(5):706-10.

38. La Mantia L, Vacchi L, Di Pietrantonj C, Ebers G, Rovaris M, Fredrikson $\mathrm{S}$, et al. Interferon beta for secondary progressive multiple sclerosis. Cochrane Database Syst Rev. 2012;1:CD005181. 


\section{Authors and Affiliations}

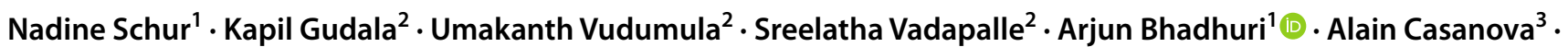
Nicholas Adlard ${ }^{4} \cdot$ Matthias Schwenkglenks ${ }^{1}$

Arjun Bhadhuri

arjun.bhadhuri@unibas.ch

Nadine Schur

nadine.schur@unibas.ch

Kapil Gudala

kapil.gudala@novartis.com

Umakanth Vudumula

umakanth.vudumula@novartis.com

Sreelatha Vadapalle

sreelatha2204@gmail.com

Alain Casanova

alain.casanova@novartis.com
Nicholas Adlard

nicholas.adlard@novartis.com

Matthias Schwenkglenks

m.schwenkglenks@unibas.ch

University of Basel, Basel, Switzerland

2 Novartis Healthcare Private Limited, Hyderabad, India

3 Novartis Pharma Schweiz AG, Rotkreuz, Switzerland

4 Novartis Pharma AG, Basel, Switzerland 